\title{
12 COMPORTAMENTO DOS ENFERMEIROS PERANTE ATOS DE BULLYING NO TRABALHO
}

\author{
| Antónia Teixeira'; ${ }^{1}$ Teresa Ferreira ${ }^{2}$; Elisabete Borges ${ }^{3}$ |
}

\section{RESUMO}

CONTEXTO: O ser humano passa grande parte da sua vida em contexto de trabalho, pelo que a sua qualidade de vida depende, entre outros fatores, das condições do local de trabalho. Comportamentos de violência, nomeadamente de bullying, são uma realidade nas organizações de saúde. A investigação tem demonstrado que os enfermeiros vivenciam este fenómeno com repercussões a nível individual, organizacional e social.

OBJETIVO: Descrever o comportamento dos enfermeiros perante atos de bullying no trabalho, enquanto vítimas e enquanto testemunhas. METODOLOGIA: Estudo de natureza qualitativa. Foram realizadas entrevistas semiestruturadas a 12 enfermeiros, cuja seleção foi efetuada através da técnica de amostragem por redes. A informação foi submetida a uma análise categorial temática segundo Bardin (2009).

RESULTADOS: Os participantes que se identificaram como vítimas referiram os seguintes comportamentos, quando sujeitos a atos de bullying: confrontar o agressor, ignorar, suporte informativo, suporte emocional e medicação antidepressiva. Aconselhar e apoiar a vítima, advogar junto do agressor, desvalorizar e passividade foram os comportamentos descritos pelos participantes que testemunharam atos de bullying. CONCLUSÕES: Os atos de bullying mostram ter um impacto a nível emocional das vítimas, sendo referida a procura de suporte emocional e o recurso a medicação antidepressiva. Consideramos que a consciencialização deste fenómeno, das suas implicações e a capacitação dos enfermeiros para a atuação em situações de atos de bullying, pode ser um fator protetor e promotor de ambientes de trabalho saudáveis.

PALAVRAS-CHAVE: Bullying; Enfermeiros; Saúde ocupacional

\section{RESUMEN}

"Comportamiento de los enfermeros ante actos de acoso laboral"

CONTEXTO: El ser humano pasa gran parte de su vida en el lugar de trabajo, por lo que su calidad de vida depende, entre otros factores, de las condiciones del lugar de trabajo. Comportamientos de violencia, incluido el acoso, son una realidad en las organizaciones de salud. La investigación ha demostrado que los enfermeros experimentan este fenómeno con consecuencias a nivel individual, organizacional y social.

OBJETIVO: Describir el comportamiento de los enfermeros ante actos de acoso, como víctimas y como testigos.

METODOLOGÍA: Estudio cualitativo. Las entrevistas semiestructuradas se llevaron a cabo con 12 enfermeros, cuya selección se hizo a través de la técnica de muestreo por redes. La información fue entregada a un análisis de las categorías temáticas según Bardin (2009). RESULTADOS: Los participantes que se identificaron como víctimas reportaron los siguientes comportamientos cuando son sometidos a actos de acoso: confrontar al agresor, ignorar, apoyo informativo, apoyo emocional y medicación antidepresiva. Asesorar y apoyar a la víctima, abogar por la víctima con el agresor, devaluar y la pasividad eran los comportamientos descritos por los participantes que fueron testigos de actos de acoso.

CONCLUSIONES: Los actos de acoso revelan tener un impacto en el nivel emocional de las víctimas, mencionando la búsqueda de apoyo emocional y el uso de medicación antidepresiva. Consideramos que la toma de conciencia de este fenómeno, sus implicaciones y la formación de los enfermeros para actuar en situaciones de acoso pueden ser un factor protector y promotor de ambientes de trabajo saludables.

DESCRIPTORES: Violencia laboral; Enfermeros; Salud laboral

\begin{abstract}
"Behavior of nurses facing bullying at work"

BACKGROUND: The human being spends a large part of his or her life in a working context, so that his/her quality of life depends on the working environment, among other factors. Behaviors of violence, particularly bullying, are a reality in healthcare organizations. Research has shown that nurses experience this phenomenon with repercussions at the individual, organizational and social level.

AIM: To describe the behavior of nurses in relation to acts of bullying at workplace, as victims and witnesses.

METHODS: A qualitative study. Through the network sampling technique, semi-structured interviews were conducted to 12 nurses. The information was submitted to a categorical thematic analysis according to Bardin (2009).

RESULTS: Participants who identified themselves as victims reported the following behaviors, when subjected to acts of bullying: confronting the aggressor, ignoring, information support, emotional support and antidepressant medication. Advising and supporting the victim, advocating with the aggressor, devaluing and passivity, were the behaviors described by participants who witnessed acts of bullying.

CONCLUSIONS: The acts of bullying have proven to have an emotional impact on the victims, being mentioned the search for emotional support and the use of antidepressant medication. We consider that the awareness of this phenomenon, its implications and the training of nurses to act in situations of bullying can be a protective factor and as well as a promoter of healthy working environments.
\end{abstract}

\section{KEYWORDS: Bullying; Nurses; Occupational health}

Submetido em 31-01-2017

Aceite em 20-06-2017

\footnotetext{
1 Mestre em Enfermagem Comunitária; Enfermeira; Centro Hospitalar São João, Alameda Prof. Hernâni Monteiro, 4200- 451 Porto, Portugal, u009637@hsjoao.min-saude.pt 2 Doutora em Psicologia da Saúde; Professora Coordenadora na Escola Superior de Enfermagem do Porto, 4200-072 Porto, Portugal, teresarodrigues@esenf.pt 3 Doutora em Enfermagem; Professora Adjunta na Escola Superior de Enfermagem do Porto, 4200-072 Porto, Portugal, elisabete@esenf.pt
}

Citação: Teixeira, A., Ferreira, T., \& Borges, E. (2017). Comportamento dos enfermeiros perante atos de bullying no trabalho. Revista Portuguesa de Enfermagem de Saúde Mental (Spe. 5), 69-73 


\section{INTRODUÇÃO}

A qualidade de vida no trabalho é um princípio básico para a realização pessoal e profissional. A violência é um dos riscos psicossociais emergentes no local de trabalho (European Agency for Safety and Health at Work [EU-OSHA], 2007; Borges e Ferreira, 2015; Neto, 2015). Enquanto um tipo de violência, o bullying caracteriza-se pela vitimização, persistente e sistemática, de um colega ou um subordinado, com o uso repetido de vários tipos de comportamentos agressivos, durante um longo período de tempo e numa situação em que as vítimas têm dificuldade em se defender (Einarsen, Hoel, Zapf, \& Cooper, 2011). Outros termos, como mobbing ou assédio moral, têm sido usados para descrever a violência em ambiente de trabalho que se apresenta de forma repetida e continuada no tempo (EU-OSHA, 2007).

O bullying é uma problemática que está presente nas instituições de saúde, de forma significativa, mas que é pouco notificado por diversos motivos, como o receio de ser rotulado de conflituoso e pensar que nada mudará ao notificar (Carter et. al, 2013). Um dos contextos de trabalho, onde o bullying tem sido investigado, é o dos enfermeiros. Este fenómeno, de interesse crescente, tem sido associado a graves consequências nos profissionais, utentes e organizações (Purpora, Cooper, \& Sharifi, 2015). O bullying afeta negativamente os enfermeiros e revela uma forte associação ao burnout, pelo que a sua prevenção é crucial (Allen, Holland, \& Reynolds, 2015). Nielsen, Magerøy, Gjerstad e Einarsen (2014) verificaram uma associação entre a exposição ao bullying e problemas a nível da saúde mental.

A realização deste trabalho tem como objetivo descrever o comportamento dos enfermeiros perante atos de bullying no trabalho, enquanto vítimas e enquanto testemunhas. É um estudo de natureza qualitativa, exploratório, descritivo e transversal, cuja finalidade é sensibilizar os enfermeiros para esta temática e promover a sua saúde no trabalho, através da consciencialização desta problemática.

\section{METODOLOGIA}

A realização deste trabalho teve como objetivo descrever o comportamento dos enfermeiros perante atos de bullying no trabalho, enquanto vítimas e enquanto testemunhas. É um estudo exploratório, descritivo e transversal, de natureza qualitativa.
Foram realizadas entrevistas semiestruturadas a 12 participantes que exerciam funções de Enfermagem há, pelo menos, 10 anos, selecionados através do método de amostragem por redes. Foi solicitado o consentimento informado aos participantes e as entrevistas foram realizadas de acordo com a disponibilidade dos participantes e em local privado. A informação foi sujeita a uma análise de conteúdo categorial de Bardin (2009).

Os participantes são maioritariamente do sexo feminino (83,3\%), com idades compreendidas entre os 31 e os 49 anos, sendo a média de 35,9 anos. Quanto ao estado civil, $50 \%$ dos participantes são solteiros e 50\% são casados. O tempo médio de exercício de funções como enfermeiro é de 13,7 anos e somente um dos participantes $(8,3 \%)$ exerce funções, no serviço atual, há menos de um ano. Sete dos participantes (58,3\%) detêm o Curso de Licenciatura em Enfermagem e cinco $(41,7 \%)$ frequentaram formação pós-graduada. A maioria dos participantes $(83,3 \%)$ trabalha em contexto hospitalar. A categoria de enfermeiro é comum a todos os participantes.

\section{RESULTADOS}

Dos 12 participantes, oito referiram conhecer alguém que já tenha vivenciado situações de bullying no trabalho. No entanto, dois casos não foram considerados para a descrição das vivências porque foram referenciados como casos únicos ou esporádicos, como mostram as seguintes unidades de registo: “... um familiar de um doente deu um empurrão à colega..." (E4); “... todos os colegas acabaram, por numa ou outra situação... sentir-se pressionados relativamente a alguns doentes..." (E5).

Relativamente às vivências enquanto vítima, seis participantes consideram que já foram vítimas de bullying no trabalho. Porém, um dos participantes referiu que “... nem numa situação nem noutra eu acho que foi uma situação persistente no tempo... não sei se se pode considerar bullying, se calhar não..." (E2). Esta descrição não foi considerada como caso de bullying, bem como a descrição de situações pontuais relatadas por outro dos participantes. Desta forma, para as vivências como vítima, consideraram-se os atos de bullying descritos por quatro participantes. 


\section{DISCUSSÃO}

\section{Comportamento das Vítimas Perante Atos de Bul- lying}

Perante os atos de bullying a que foram sujeitos, os participantes referiram que, confrontar o agressor, ignorar, suporte informativo, suporte emocional e medicação antidepressiva, foram os comportamentos adotados, como ilustra a tabela n. ${ }^{\circ} 1$.

Tabela 1 - Comportamento das vítimas perante atos de bullying

\begin{tabular}{|c|c|}
\hline \multicolumn{2}{|c|}{$\begin{array}{l}\text { CATEGORIA - COMPORTAMENTO DAS VÍTIMAS } \\
\text { PERANTE ATOS DE BULLYING }\end{array}$} \\
\hline SUBCATEGORIA & UNIDADES DE REGISTO \\
\hline Confrontação & $\begin{array}{l}\text { “... às vezes a situação em si dá para } \\
\text { confrontar a pessoa..." (E1) }\end{array}$ \\
\hline Ignorar & $\begin{array}{l}\text { “... às vezes acabo mesmo por ignorar } \\
\text { um bocadinho os comportamentos } \\
\text { delas...”(E1) } \\
\text { “... as pessoas falavam e eu ignorava..." } \\
\text { (E11) }\end{array}$ \\
\hline Suporte informativo & $\begin{array}{l}\text { “... procurar informação...” (E7) } \\
\text { “... procurava perceber a melhor forma } \\
\text { de fazer as coisas... tentar, junto de } \\
\text { outros colegas... saber como é que eles } \\
\text { faziam para tentar saber se eu real- } \\
\text { mente fazia assim tão mal...” (E11) }\end{array}$ \\
\hline Suporte emocional & $\begin{array}{l}\text { “... Éramos várias, apoiávamo-nos } \\
\text { umas às outras...” (E9) } \\
\text { “... do resto da equipa fui muito bem } \\
\text { recebida...” (E9) } \\
\text { “... valia a pena pela parte dos doentes, } \\
\text { e pela parte de alguns colegas que... } \\
\text { foram capazes de me reconhecer algum } \\
\text { valor, mesmo apesar de estar a começar } \\
\text { a trabalhar..." (E11) } \\
\text { “... é um bocado refugiar na família...” } \\
\text { (E11) }\end{array}$ \\
\hline $\begin{array}{l}\text { Medicação } \\
\text { antidepressiva }\end{array}$ & $\begin{array}{l}\text { “... fiz medicação antidepressiva... pre- } \\
\text { cisei de fazer durante uns meses..." (E7) }\end{array}$ \\
\hline
\end{tabular}

Confrontar o agressor foi mencionado por um dos participantes (E1). Comunicar ao agressor, que o seu comportamento é inaceitável, é um dos desafios para os gestores, quando tomam conhecimento de comportamentos de bullying nas suas equipas (Oade, 2009). Existem diferentes tipologias de agressores; uns são capazes de aceitar feedback, pedir desculpas e tomar medidas para mudar o seu comportamento, enquanto outros sentem satisfação ao infligir dor e sofrimento aos outros (McCulloch, 2010).

Ignorar também foi um comportamento que emergiu dos relatos dos participantes (E1, E11).
Segundo Hirigoyen (2002), as consequências a nível psíquico tornam-se mais graves quando a pessoa toma consciência de que existe uma intenção de a magoar. Os dados obtidos neste estudo não permitem aferir se os participantes, para além de ignorarem os comportamentos do agressor, ignoraram a intencionalidade de serem prejudicados.

A procura de suporte informativo foi outro comportamento referido (E7, E11). No caso de um dos participantes (E7), a necessidade de procura de informação resultou de uma mudança de serviço, de forma a colmatar a falta de apoio sentida quando ingressou no novo serviço. No outro caso (E11), o participante menciona que essa necessidade surgiu das críticas constantes ao seu trabalho, sendo esta uma estratégia para comprovar se as críticas tinham ou não fundamento. Este dado vai ao encontro da investigação de Hallberg e Strandmark (2006), onde a vítima luta pela confirmação de que o bullying foi injusto e não foi por sua culpa.

Os participantes referiram ter recorrido ao suporte emocional, nomeadamente ao apoio de colegas e familiares. Uma participante (E9) descreve o apoio mútuo com outras colegas, que ingressaram no serviço juntamente com ela e que passaram pelo mesmo processo de integração dificultadora, evidenciando a ideia de partilha de experiências e de suporte pelos pares. Os familiares e amigos podem dar um valioso contributo no apoio às vítimas (Oade, 2009). Hirigoyen (2002) verificou, no seu inquérito, que $39 \%$ das vítimas de assédio moral procurou ajuda junto de colegas e que a gravidade das consequências para a saúde depende da vulnerabilidade da vítima, onde se inclui a falta de apoio familiar ou de amigos. $\mathrm{O}$ apoio social, onde se inclui o apoio emocional, contribui para a saúde e o bem-estar das pessoas (Borges e Ferreira, 2013; Serra, 2011). Borges e Ferreira (2015) constataram que um maior recurso ao suporte social conduziu à perceção de melhor qualidade de vida. O suporte emocional pode ser, deste modo, um fator protetor para as vítimas.

O recurso a medicação antidepressiva foi referido por um participante (E7). Vartia (2001) verificou que as vítimas de bullying recorriam mais vezes a indutores do sono e sedativos do que as pessoas que não eram vítimas deste tipo de violência. $\mathrm{O}$ bullying contribui substancialmente para estados de ansiedade e depressão, afetando a satisfação no trabalho (Hauge, Skogstad, \& Einarsen, 2010). 


\section{Comportamento das Testemunhas Perante Atos de Bullying}

Aconselhar e apoiar a vítima, advogar junto do agressor, desvalorizar e passividade foram os comportamentos descritos pelos participantes que testemunharam atos de bullying, como se ilustra na tabela $n .^{\circ} 2$.

Tabela 2 - Comportamento das testemunhas perante atos de bullying

\begin{tabular}{|c|c|}
\hline \multicolumn{2}{|c|}{$\begin{array}{l}\text { CATEGORIA - COMPORTAMENTO DAS TESTE- } \\
\text { MUNHAS PERANTE ATOS DE BULLYING }\end{array}$} \\
\hline SUBCATEGORIA & UNIDADES DE REGISTO \\
\hline $\begin{array}{l}\text { Aconselhar a } \\
\text { vítima }\end{array}$ & $\begin{array}{l}\text { “... tentar arranjar com ela... me- } \\
\text { canismos de defesa para não se deixar } \\
\text { afetar..." (E1) } \\
\text { “... arranjar uma estratégia para tentar } \\
\text { mudar isso mesmo... mas ser ela própria } \\
\text { a tomar essa decisão...” (E3) }\end{array}$ \\
\hline Apoiar a vítima & $\begin{array}{l}\text { “... Eu fui sempre um ouvinte dessa pes- } \\
\text { soa... tentar compreendê-la, confortá- } \\
\text { la... dar-lhe apoio..." (E3) }\end{array}$ \\
\hline $\begin{array}{l}\text { Advogar junto do } \\
\text { agressor }\end{array}$ & $\begin{array}{l}\text { “... dizer que exagera...” (E1) } \\
\text { “... eu tentava sempre demonstrar que as } \\
\text { pessoas... que são recém-formadas, não } \\
\text { podem ter os mesmos conhecimentos... } \\
\text { (E12) }\end{array}$ \\
\hline Desvalorizar & $\begin{array}{l}\text { “... tentar desvalorizar... porque nor- } \\
\text { malmente este tipo de pessoas... não } \\
\text { aceitam..." (E1) }\end{array}$ \\
\hline Passividade & $\begin{array}{l}\text { “... Não foi nenhuma, não foi nada... } \\
\text { uma pessoa também acaba por querer-se } \\
\text { resguardar um bocadinho...” (E6) }\end{array}$ \\
\hline
\end{tabular}

Aconselhar e apoiar a vítima de bullying foram comportamentos referidos pelas testemunhas. Domínguez, Gil-Monte e Devis (2011) identificaram que, quanto maior o apoio social no trabalho, menor o bullying sofrido pelo trabalhador. $\mathrm{O}$ aconselhamento permite conter o sofrimento das pessoas envolvidas no fenómeno de bullying (Tehrani, 2011). Perante o agressor, advogar e desvalorizar foram os comportamentos mencionados. Ao advogar junto do agressor, a testemunha poderá promover a tomada de consciência do agressor relativamente aos seus atos. Desvalorizar foi referido por um participante (E1), que deixa presente a noção de que é difícil o agressor aceitar que se lhe diga algo relativamente à situação. Um dos participantes mencionou que não tomou nenhuma atitude, fundamentando que “... uma pessoa também acaba por quererse resguardar um bocadinho...” (E6). Uma das causas mais apontadas pelos enfermeiros que experienciaram condutas de mobbing, no estudo de Carvalho (2007), foi a solidariedade com os outros colegas e não virar as costas à injustiça. O receio de ser prejudicado pode ter contribuído para esta atitude de passividade.

\section{CONCLUSÃO}

Este estudo teve como objetivo descrever o comportamento dos enfermeiros perante atos de bullying no trabalho, enquanto vítimas e enquanto testemunhas. Os participantes que se identificaram como vítimas referiram os seguintes comportamentos: confrontar o agressor, ignorar, suporte informativo, suporte emocional e medicação antidepressiva. Aconselhar e apoiar a vítima, advogar junto do agressor, desvalorizar e passividade foram os comportamentos descritos pelas testemunhas de atos de bullying.

Podemos concluir que os atos de bullying têm impacto a nível emocional das vítimas, na medida em que houve procura de suporte emocional e recurso a medicação antidepressiva.

Nenhum dos participantes referiu ter denunciado os atos de bullying. Consideramos necessária mais investigação para se compreender o motivo pelo qual não foram efetuadas denúncias. $\mathrm{O}$ comportamento humano depende de vários fatores. A informação sobre o fenómeno poderá ser um dos fatores que contribui para o comportamento das pessoas envolvidas.

\section{IMPLICAÇÕES PARA A PRÁTICA CLÍNICA}

Consideramos que a consciencialização deste fenómeno, e das suas implicações, pode ser um fator importante no comportamento das pessoas envolvidas, pelo que a sua divulgação, junto dos profissionais e dos estudantes de Enfermagem, se torna fundamental. Salientamos também a importância da capacitação dos enfermeiros para a atuação em situações de atos de bullying. É essencial o desenvolvimento de planos de atuação, que incluam suporte emocional, e, essencialmente, projetos de prevenção do bullying. A sua prevenção torna-se essencial para contextos de trabalho mais saudáveis para os profissionais e mais seguros para a população que recebe os seus cuidados.

\section{REFERÊNCIAS BIBLIOGRÁFICAS}

Allen B. C., Holland P., \& Reynolds, R. (2015). The effect of bullying on burnout in nurses: the moderating role of psychological detachment. Journal of Advanced Nursing 71(2), 381-390. doi: 10.1111/jan.12489

Bardin, L. (2009). Análise de conteúdo (5ª ed.). Lisboa: Edições 70. (Tradução do original em francês L'analyse de contenu. S.l.: Presses Universitaires de France, 1977). 
Borges, E., \& Ferreira, T. (2015). Bullying no trabalho: Adaptação do Negative Acts Questionnaire-Revised (NAQ-R) em enfermeiros. Revista Portuguesa de Enfermagem de Saúde Mental (13), 25-33.

Borges, E., \& Ferreira, T. (2013). Relaxamento: Estratégia de intervenção no stress. Revista Portuguesa de Enfermagem de Saúde Mental (10), 37-42.

Carter, M., Thompson, N., Crampton, P., Morrow, G., Burford, B., Gray, C., \& Illing, J. (2013). Workplace bullying in the UK NHS: a questionnaire and interview study on prevalence, impact and barriers to reporting. BMJ Open, 3 (6), e002628. doi: 10.1136/bmjopen-2013-002628

Carvalho, G. D. (2007). Mobbing: Assédio moral em contexto de enfermagem: estudo preliminar. Revista Investigação em Enfermagem, (15), 43-55. Acedido em: http://hdl.handle.net/10198/5331

Domínguez, N., Gil-Monte, P., \& Devis, J. (2011). Antecedentes y consecuencias del acoso psicológico en el trabajo. Psicotherma, 23 (4), 617-623. Acedido em http://www.psicothema.com/pdf/3931.pdf

Einarsen, S., Hoel, H., Zapf, D., \& Cooper, C. L. (2011). The concept of bullying and harassment at work: The European tradition. In S. Einarsen, H. Hoel, D. Zapf, \& C. L. Cooper (Eds), Bullying and harassment in the workplace: Developments in theory, research, and practice (pp. 3-39). (2th ed.). Boca Raton, FL: CRC Press.

European Agency for Safety and Health at Work (2007). Expert forecast on emerging psychosocial risks related to occupational safety and health. Luxembourg. Acedido em: https://osha.europa.eu/en/toolsand-publications/publications/reports/7807118

Hallberg, L., \& Strandmark, M. (2006). Health consequences of workplace bullying: Experiences from the perspective of employees in the public service sector. International Journal of Qualitative Studies on Health and Well-being (1), 109-119. doi: $10.1080 / 17482620600555664$

Hauge, L., Skogstad, A., \& Einarsen, S. (2010). The relative impact of workplace bullying as a social stressor at work. Scandinavian Journal of Psychology, 51, 426433. doi: 10.1111/j.1467-9450.2010.00813.x
Hirigoyen, M. F. (2002). O assédio no trabalho: Como distinguir a verdade. Cascais: Editora Pergaminho. (Tradução do original em francês Malaise dans le travail. S.1.: Syros, 1998).

McCulloch, B. (2010). Dealing with bullying behaviours in the workplace: What works - A practitioner's view. Journal of the International Ombudsman Association, 3 (2), 39-51.

Neto, H. (2015). Estratégias organizacionais de gestão e intervenção sobre riscos psicossociais do trabalho. International Journal on Working Conditions, 9, 1-21. Acedido em http://ricot.com.pt/artigos/1/IJWC.9_ HVN.p.1-21.pdf

Nielsen, M. B., Magerøy, N., Gjerstad, J., \& Einarsen, S. (2014). Workplace Bullying and subsequent health problems. Tidsskr Nor Legeforen, 12/13(134), 12331238. doi: $10.4045 /$ tidsskr.13.0880

Oade, A. (2009). Managing workplace bullying: How to identify, respond to and manage bullying behavior in the workplace. Hampshire: Palgrave Macmillan.

Purpora, C., Cooper, A., \& Sharifi, C. (2015). The prevalence of nurses' perceived exposure to workplace bullying and its effect on nurse, patient, organization and nursing-related outcomes in clinical settings: a quantitative systematic review protocol. JBI Database of Systematic Reviews and Implementation Reports, 13 (9), 51-62. doi: 10.11124/jbisrir-2015-2225

Serra, A. V. (2011). O stress na vida de todos os dias ( $3^{a}$ ed.). Coimbra: Gráfica de Coimbra, Lda.

Tehrani, N. (2011). Workplace bullying: The role for counselling. In: S. Einarsen, H. Hoel, D. Zapf, \& C. L. Cooper (Eds), Bullying and harassment in the workplace: Developments in theory, research, and practice (pp. 381-396). (2th ed.) Boca Raton, FL: CRC Press.

Vartia, M. (2001). Consequences of workplace bullying with respect to the well-being of its targets and the observers of bullying. Scandinavian Journal of Work, Environment \& Health, 27 (1), 63-69. doi:10.5271/ sjweh.588 


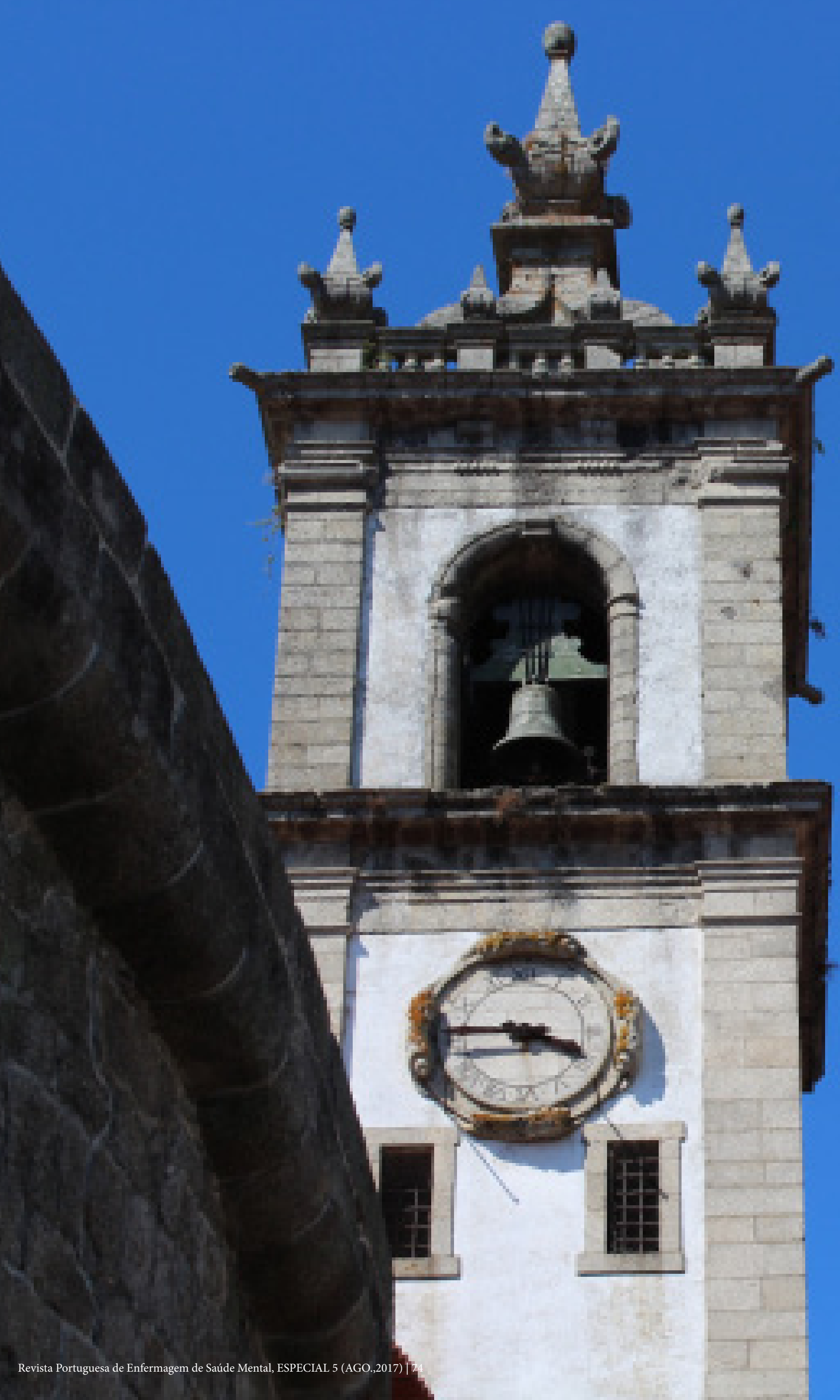

